\title{
A Three-Dimensional Model of Turbulent Core Annular Flow Regime
}

\author{
Saliha Nouri, ${ }^{1,2}$ Zouhaier Hafsia $\mathbb{D}^{1,3}$ Salah Mahmoud Boulaaras $\mathbb{D}^{10},{ }^{4,5}$ Ali Allahem $\mathbb{D}^{\mathbb{D}}{ }^{6}$ \\ Salem Alkhalaf, ${ }^{7}$ and Baowei Feng ${ }^{8}$ \\ ${ }^{1}$ Department of Physics, College of Science and Arts at ArRass, Qassim University, Buraydah, Saudi Arabia \\ ${ }^{2}$ Department of Physics, University of Tunis, Tunis, Tunisia \\ ${ }^{3}$ Department of Physics, University of Tunis El-Manar, Tunis, Tunisia \\ ${ }^{4}$ Department of Mathematics, College of Science and Arts at ArRass, Qassim University, Buraydah, Saudi Arabia \\ ${ }^{5}$ Laboratory of Fundamental and Applied Mathematics of Oran (LMFAO), University of Oran 1, Oran 31000, Algeria \\ ${ }^{6}$ Department of Mathematics, College of Science, Qassim University, Buraydah, Saudi Arabia \\ ${ }^{7}$ Department of Computer Science, College of Science and Arts at ArRass, Qassim University, Buraydah, Saudi Arabia \\ ${ }^{8}$ Department Economic Mathematics, Southwestern University of Finance and Economics, Chengdu, China
}

Correspondence should be addressed to Zouhaier Hafsia; hafsia.zouhaier@gmail.com

Received 4 October 2021; Accepted 20 October 2021; Published 31 October 2021

Academic Editor: Heng Liu

Copyright (C) 2021 Saliha Nouri et al. This is an open access article distributed under the Creative Commons Attribution License, which permits unrestricted use, distribution, and reproduction in any medium, provided the original work is properly cited.

In this study, three-dimensional (3D) turbulent core annular flow (CAF) regime is investigated numerically. The proposed model is based on the 3D Reynolds average Navier-Stokes (RANS) equations combined with a pure convective transport equation of the volume of fluid (VOF) to predict the interface between the oil and water phases. The $\mathrm{k}-\omega$ turbulence model is adopted to better reproduce the oil and water flow characteristics. The two-phase (CAF) regime can be predicted by two inlet configurations: the T-junction (3D-T) and the straight pipe (3D-S). These two configurations are simulated and compared for pipe diameter $D=$ $0.026 \mathrm{~m}$ and pipe length $L=4 \mathrm{~m}$. For these two inlet configurations, the computed mixture velocity profile and the water volume fraction at a test section $(z=100 \mathrm{D})$ were compared to experimental measurements. The 3D-T configuration gives more appropriate results. The 3D-S slightly overestimates the maximum velocity at the test section and the lower and upper water layer of the (CAF) flow is shifted in the upward direction. For the 3D-T, the relative error in the pressure drop is $3.3 \%$. However, for the $3 \mathrm{D}-\mathrm{S}$, this error is $13.0 \%$.

\section{Introduction}

Two-phase oil-water flow is a particular case of multiphase flow and has a great interest especially in the petroleum, nuclear, and chemical industries. The distribution of each phase affects the pressure drop in a more complicated way than in a single-phase flow. The main tasks of the numerical and experimental investigations are the predetermination of the characteristics of the flow regimes, water holdup, and pressure drop along the transport cylindrical pipe. Analytical solutions were restricted to the formulations of the pressure drop (Brauner in [1]). Following the prescribed superficial velocities of the two phases, there are mainly different flow regimes: stratified, bubble, dispersed, plug, slug, and core annular flow (CAF). The slug and the stratified flow have been extensively studied experimentally and numerically (Cherlo et al., Desir et al., and Al-Yaari and Abu-Sharkh in [2-4]). Recently, the CAF regime has become the more interesting way for the transportation of oil.

The experimental measurements are the primal method to study the distribution of the oil and water phases under different superficial velocities of the two phases. Charles et al., in [5], conducted the first experimental study of oilwater flow in a uniform cross section and horizontal pipe with equal densities and low oil viscosity. In these experiments, the CAF was observed. Elseth, in [6], studied 
experimentally the pressure drop and local phase fractions of the stratified and dispersed oil and water. Amundsen, in [7], investigated experimentally the effects of the pipe inclination and water cuts on the flow regime and the pressure drop for a constant mixture velocity. Balakhrisna et al., in [8], studied experimentally the flow patterns for oil-water and kerosenewater of high viscous oil through a sudden contraction and expansion of a horizontal pipe. The contraction and expansion two-phase flow coefficients are lower than the corresponding one for a single-phase water flow. Following the experimental study of Al-Awadi in [9], the water injection direction (horizontally or vertically) through the $\mathrm{T}$-junction pipe does not affect the flow regime and pressure gradients.

Numerical results and experimental investigations for a stratified oil-water flow in the horizontal pipe were conducted by Santos et al. in [10]. The considered pipe length in the computation is reduced compared to the experiments. Liu et al., in [11], validated the numerical model based on the volume of fraction (VOF) in the case of the oilwater flow through an elbow system for upward, downward, and horizontal configurations. Shi, in [12], conducted an experimental and numerical study of highviscosity oil-water flow in horizontal pipes for two diameters. A T-junction cylindrical pipe was considered to predict the (CAF) regime. Due to the higher viscosity of the oil phase, a laminar parabolic profile was specified for the oil inlet at the branch pipe and a turbulent power profile was imposed for the water inlet at the main pipe. The SST $\mathrm{k}-\omega$ turbulence model with turbulence damping at the interface leads to more accurate results. Al-Jadidi, in [13], conducted a series of numerical (CAF) flow using the large Eddy simulation (LES) model coupled with the VOF mixture model to simulate the heavy oil and water flow regimes. Konings, in [14], imposed the oil and the water inlet velocities at the same cross-section inlet to reproduce the CAF flow under periodic boundaries conditions at the inlet and the outlet. Jiang et al., in [15], studied numerically the CAF regime using a T-junction pipe. Ayuba et al., in [16], studied numerically the CAF flow using lateral and central injections of water and oil, respectively, through a horizontal pipe with a uniform cross section.

In the previous numerical studies, the CAF is generated by different ways, but the comparison between the different approaches is not analyzed. The objective of the present study is to investigate the CAF by two methods: through a $\mathrm{T}$-junction pipe (3D-T) and through a straight pipe (3D-S). The principal recommendations corresponding to the accuracy of the CAF regime generation is then deduced.

\section{Problem Description}

Figure 1 represents the two geometric configurations usually adopted to generate the (CAF) regime. The $\mathrm{T}$-junction configuration is formed by two circular pipes. The main and the branch pipe having the same diameter as indicated by the experimental conducted by Charles et al. in [5] which is $D=0.026 \mathrm{~m}$. The length of the pipe upstream the junction is $B=0.2 \mathrm{~m}$ and downstream the junction is $L=4 \mathrm{~m}$ sufficient to obtain the developed flow. The length of the branch pipe is equal to $B$. In the configuration of a T-junction (3D-T), the oil enters at the inlet of the branch pipe (OI) and the water is injected at the inlet of the main pipe (WI). In the second configuration of a straight pipe (3D-S), the water is injected circumferentially at the same cross section as the core from the oil inlet. The test section is located at $z=100$ and $D=$ $2.6 \mathrm{~m}$ from the pipe inlet as considered by Shi in [12]. In all simulated cases, a uniform gravity field is imposed equal to $g_{y}=-9.81 \mathrm{~m} / \mathrm{s}^{2}$.

In this case, the inner radius pipe $R_{1}$ is given by the following equation (Konings) in [14]:

$$
R_{1}=\frac{R_{2}}{\sqrt{\left(U_{\mathrm{sw}} / U_{\mathrm{so}}\right) h+1}},
$$

where $R_{2}$ is the outer radius pipe $\left(R_{2}=D / 2=0.013 \mathrm{~m}\right), U_{\text {sw }}$ is the superficial velocity of water, $U_{\text {so }}$ is the superficial velocity of oil, and $h$ is the holdup ratio $(h=1.39)$.

This gives $R_{1}=0.0084 \mathrm{~m}$ for $U_{\mathrm{sw}}=U_{\text {so }}=0.55 \mathrm{~m} / \mathrm{s}$ which correspond to a mixture velocity $U_{m}=U_{\text {so }}+U_{\text {sw }}=1.1 \mathrm{~m} / \mathrm{s}$ and an inlet water cut, $C_{w}=0.5$.

The fluid density and dynamic viscosity of each phase are

(i) For oil phase: $\rho_{o}=997.2 \mathrm{Kg} / \mathrm{m}^{3}$ and $\mu_{o}=0.0168 \mathrm{~Pa} . \mathrm{s}$

(ii) For water phase: $\rho_{w}=998 \mathrm{Kg} / \mathrm{m}^{3}$ and $\mu_{w}=0.001 \mathrm{~Pa} . \mathrm{s}$

Based on these fluid characteristics, the corresponding Reynolds numbers for oil and water phases are, respectively,

$$
\begin{aligned}
& \operatorname{Re}_{\text {so }}=\frac{\rho_{o} U_{\text {so }} D}{\mu_{o}}=848.8, \\
& \operatorname{Re}_{\text {sw }}=\frac{\rho_{o} U_{\text {sw }} D}{\mu_{w}}=14271.4 .
\end{aligned}
$$

Following these values, the turbulent flow model is needed for the two-phase mixture.

\section{Mathematical Model}

3.1. Governing Transport Equations. In the mixture model, the same Reynolds average Navier-Stokes (RANS) equations are used for the two immiscible phases constituted by water and oil. These equations can be written in the following form (Shi et al.) in [17]:

The average mass conservation:

$$
\frac{\partial \rho}{\partial t}+\frac{\partial}{\partial x_{j}}\left(\rho U_{j}\right)=0, \quad j=1,2,3 .
$$

The average momentum conservation equations"

$$
\begin{aligned}
\frac{\partial}{\partial t}\left(\rho U_{i}\right)+\frac{\partial}{\partial x_{j}}\left(\rho U_{j} U_{i}\right)= & -\frac{\partial P}{\partial x_{i}}+\frac{\partial}{\partial x_{j}}\left[\left(\mu+\mu_{t}\right) \frac{\partial U_{i}}{\partial x_{j}}\right] \\
& +g_{i}+F_{i}, \quad i, j=1,2,3
\end{aligned}
$$




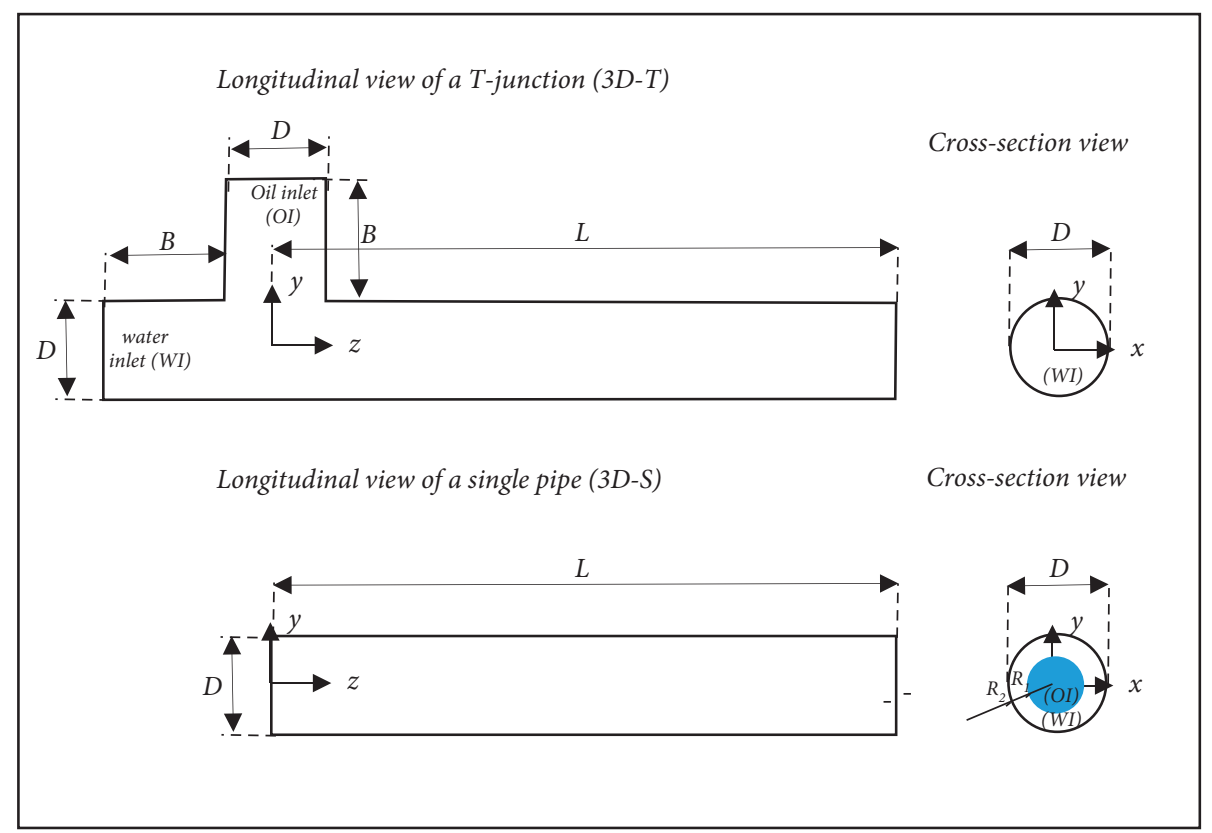

Figure 1: Sketch of 3D geometries of the T-junction (3D-T) and a straight horizontal pipe (3D-S) for a (CAF) flow regime.

where $\rho$ is the mixture density and $\mu$ is the mixture dynamic molecular viscosity given by

$$
\begin{aligned}
& \rho=\rho_{1} \alpha_{1}+\rho_{2} \alpha_{2}, \\
& \mu=\mu_{1} \alpha_{1}+\mu_{2} \alpha_{2},
\end{aligned}
$$

where $\rho_{o}$ and $\rho_{w}$ are, respectively, the density of the oil and water, $\alpha_{o}$ and $\alpha_{w}$ are the volume fraction of each phase such that $\alpha_{o}+\alpha_{w}=1, U_{i}$ are the mean velocity components, $P$ is mean pressure, $\mu_{t}$ is the dynamic turbulent viscosity, $g_{i}$ is the gravity acceleration field, and $F_{i}$ are the components of external force per unit volume associated with the surface tension.

The $k$ - $\omega$ model with shear stress transport is adopted as the turbulence model. Following this model, the turbulent viscosity is defined as (Wilcox) in [18]:

$$
\mu_{t}=f_{\mu} \frac{\rho k}{\omega}
$$

The turbulent kinetic energy $(k)$ transport equation:

$$
\begin{aligned}
\frac{\partial}{\partial t}(\rho k)+\frac{\partial}{\partial x_{j}}\left(\rho k U_{j}\right)= & \frac{\partial}{\partial x_{j}}\left[\left(\mu+\frac{\mu_{t}}{\sigma_{k}}\right) \frac{\partial k}{\partial x_{j}}\right] \\
& +\rho\left(P_{k}-f_{2} \varepsilon\right) .
\end{aligned}
$$

The specific dissipation rate $(\omega)$ transport equation:

$$
\begin{aligned}
\frac{\partial}{\partial t}(\rho \omega)+\frac{\partial}{\partial x_{j}}\left(\rho \omega U_{j}\right)= & \frac{\partial}{\partial x_{j}}\left[\left(\mu+\frac{\mu_{t}}{\sigma_{\omega}}\right) \frac{\partial \omega}{\partial x_{j}}\right] \\
& +\rho \omega\left(f_{1} C_{1 \omega} \frac{P_{k}}{k}-C_{2 \omega} \omega\right),
\end{aligned}
$$

where $P_{k}$ is the volumetric production rate of $(k)$ :

$$
P_{k}=\frac{\mu_{t}}{\rho}\left(\frac{\partial U_{i}}{\partial x_{j}}+\frac{\partial U_{j}}{\partial x_{i}}\right) \frac{\partial U_{i}}{\partial x_{j}} .
$$

$f_{\mu}, f_{1}$, and $f_{2}$ are low Reynolds damping functions. The turbulent dissipation rate $(\varepsilon)$ is given by

$$
\varepsilon=C_{D} \omega k \text {. }
$$

The following constants are commonly used:

$$
\begin{aligned}
\sigma_{k} & =2.0, \\
\sigma_{\omega} & =2, \\
C_{D} & =0.09, \\
C_{1 \omega} & =\frac{5}{9}, \\
C_{2 \omega} & =\frac{3}{40} .
\end{aligned}
$$

At high Reynolds numbers, the damping functions are set to unity and given by

$$
\begin{aligned}
& f_{\mu}=\frac{(1 / 40)+\left(R_{e, t} / R_{k}\right)}{1+\left(R_{e, t} / R_{k}\right)}, \quad R_{k}=6.0, \\
& f_{1}=\frac{1}{f_{\mu}} \frac{0.1+\left(R_{e, t} / R_{\omega}\right)}{1+\left(R_{e, t} / R_{\omega}\right)}, \quad R_{\omega}=2.7, \\
& f_{2}=\frac{(5 / 18)+\left(R_{e, t} / R_{B}\right)^{4}}{1+\left(R_{e, t} / R_{B}\right)^{4}}, \quad R_{B}=8.0,
\end{aligned}
$$

where $R_{e, t}$ is the turbulent Reynolds number: 


$$
R_{e, t}=\frac{\rho k}{\mu \omega} .
$$

At high Reynolds numbers, the damping functions $f_{\mu}$, $f_{1}$, and $f_{2}$ tend to unity.

According to Brackbill et al., in [19], the surface tension at the interface between oil and water is transformed to a continuum surface force (CSF). Following this model, the surface tension force is proportional to the curvature $\kappa$ :

$$
\vec{F}=\sigma \kappa \frac{\rho \vec{\nabla} \alpha_{1}}{1 / 2\left(\rho_{1}+\rho_{2}\right)},
$$

where $\kappa$ is the interface curvature defined in terms of the divergence of the unit normal as

$$
\kappa=\vec{\nabla} \cdot\left(\frac{\vec{n}}{\bar{n}}\right) .
$$

The surface normal is defined as the gradient of the volume fraction of the oil phase:

$$
\vec{n}=\vec{\nabla} \alpha_{1}
$$

For the adopted validation case investigated experimentally by Charles et al. in [5], the surface tension between the oil and water phases is $\sigma=0.045 \mathrm{~N} / \mathrm{m}$. An academic version of a computational fluid dynamics code was used to solve these transport equations and the associated initial and boundaries conditions.

3.2. Initial and Boundary Conditions. At the initial time of simulation $(t=0 \mathrm{~s})$, the horizontal pipe with a uniform cross section is filled with water. At the pipe wall, a no-slip boundary condition is considered. At the outlet, zero pressure is specified. For the T-junction pipe, the oil inlet is located at the inlet of the branch pipe and a parabolic laminar profile is specified as follows:

$$
W_{o, \text { in }}=2 W_{s, o}\left(1-\frac{r_{y}^{2}}{R_{2}^{2}}\right),
$$

where $r_{y}$ is the radial distance in the $x z$-plane: $r_{y}=$ $\sqrt{x^{2}+z^{2}}$.

For a single pipe, the oil inlet is located at the same location of the water inlet and $r_{y}$ is replaced by $r_{z}=$ $\sqrt{x^{2}+y^{2}}$.

For water inlet, a turbulent power profile is imposed:

$$
W_{w, \text { in }}=K W_{s, w}\left(1-\frac{r_{z}}{R^{2}}\right)^{1 / n}
$$

where $r_{y}$ is the radial distance in the $x y$-plane and $K$ is given by the following relation (Schlichting) in [20]:

$$
K=\frac{(n+1)(2 n+1)}{2 n^{2}} .
$$

The coefficient $n$ depended on the water Reynolds number and is taken equal to $n=6.6$.
The following equations are adopted to calculate the turbulent kinetic energy and the specific dissipation rate at the inlet:

$$
\begin{aligned}
& k=(\mathrm{IU})^{2}, \\
& \omega=\frac{\varepsilon}{C_{D} k}, \\
& \varepsilon=\frac{C_{D}^{3 / 4} k^{3 / 2}}{0.1 H},
\end{aligned}
$$

where $H$ is a characteristic inlet dimension as the hydraulic radius.

3.3. Numerical Methods. For the two inlet configurations (3D-T and 3D-S), the computational domain contains 1100 $\mathrm{O}$-grids in the cross section of the pipe and 400 cells in the axial direction. Hence, a structured mesh was adopted to simulate the CAF of oil and water phases. The first cell layer in the cross section of the pipe is located at $0.2 \mathrm{~mm}$ in order to satisfy that $y^{+}<5$.

The PISO algorithm (pressure implicit with splitting of the operator) is used to solve the velocity-pressure coupling. The same residual $10^{-5}$ is adopted for all solved variables.

As indicated by Shi in [12], the geometric reconstruction (piecewise-linear) approach permits to accurately capture the interface variation between oil and water phases.

A fixed time step is chosen $\Delta t=0.01 \mathrm{~s}$. For each inlet configuration, the fully developed flow (FDF) is attained if the pressure profile along the pipe axis is unchanged with time.

\section{Validation of the Numerical Results}

The numerical results are validated for low viscosity results in terms of the mixture velocity profile and the water volume fraction for the oil-water flow. The comparative three-dimensional study between the T-junction pipe and the straight pipe is made for the mixture oil-water velocity equal to $U_{m}=1.1 \mathrm{~m} / \mathrm{s}$ and a water cut $C_{w}=0.5$.

The fully developed flow (FDF) is attained if the pressure profile along the pipe axis is unchanged with time. Figure 2 represents the pressure along the pipe axis for different times after the water phase reaches the pipe outlet and for the T-junction inlet configuration denoted 3D-T. All these profiles have the same shape except the approaching outlet region. At the junction of the main and branch pipes, there is a sudden decrease of the pressure, and further with downstream, the pressure decreases gradually. The quasisteady flow regime is attained from the time $t=5 \mathrm{~s}$. The predicted pressure drop for this CAF is $498 \mathrm{~Pa} / \mathrm{m}$ which is close to the experimental value measured by Charles et al. in [5] and equal to $482 \mathrm{~Pa} / \mathrm{m}$ corresponding to a relative error equal to $3.3 \%$. For the straight pipe (3D-S), the computational pressure profiles show that the FDF is attained at the same time and that the simulated pressure drop is $420 \mathrm{~Pa} / \mathrm{m}$ and the relative error is around $13.0 \%$. 


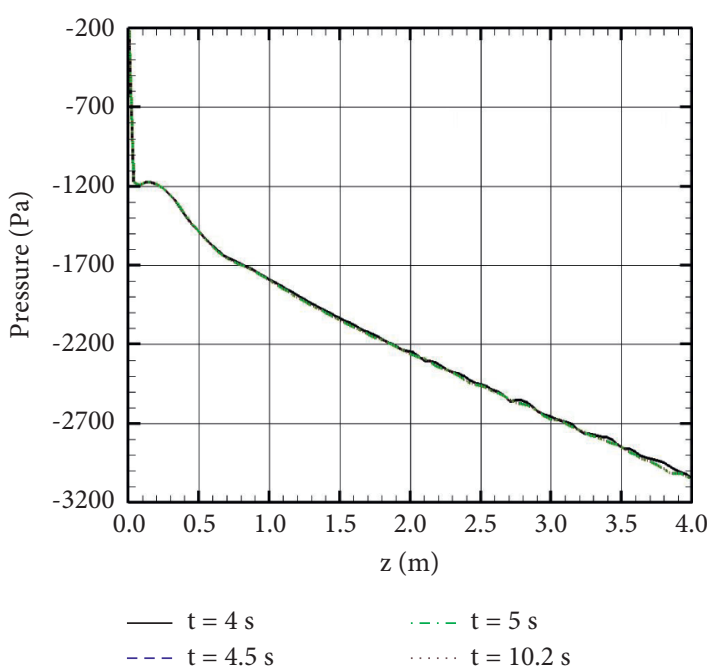

Figure 2: Pressure variation along the pipe axis for different time instants and for the 3D-T configuration.
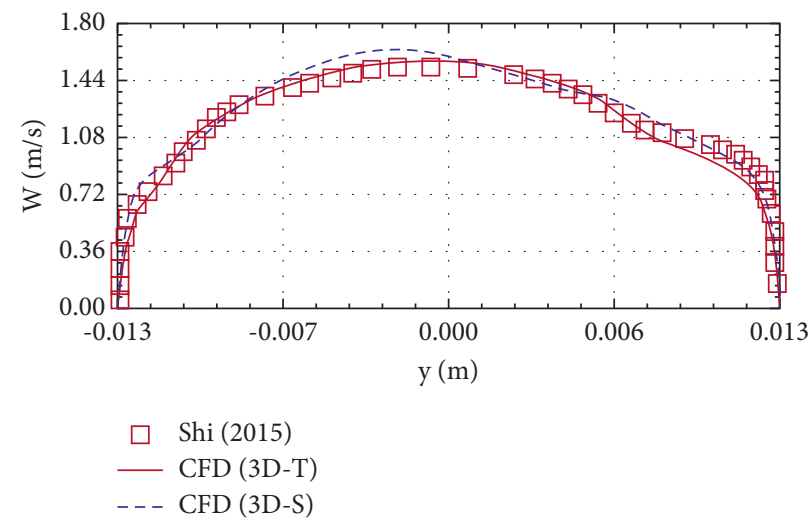

FIGURE 3: Comparison between the simulated axial velocity for mixture velocity $1.1 \mathrm{~m} / \mathrm{s}$ and the numerical results of Shi.

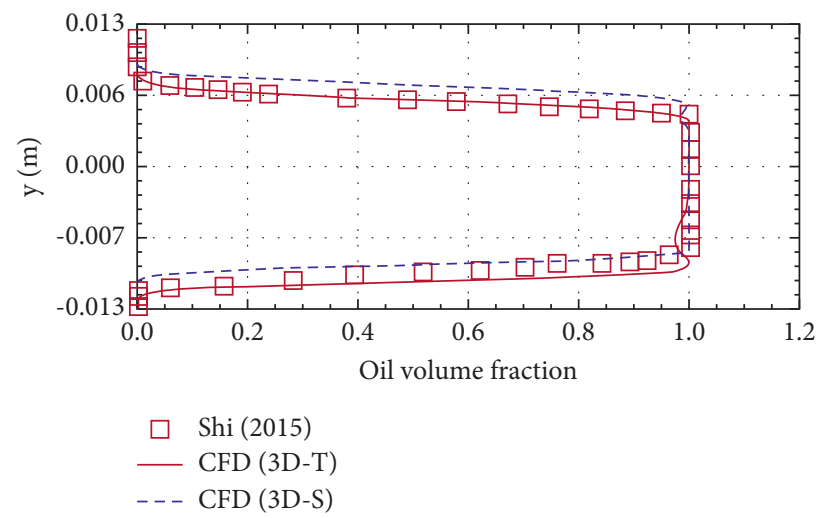

Figure 4: Comparison between the simulated oil volume fraction for mixture velocity $1.1 \mathrm{~m} / \mathrm{s}$ and the numerical results of Shi.

The simulated axial mixture velocity of CAF for the two inlet configurations denoted $3 \mathrm{D}-\mathrm{T}$ and $3 \mathrm{D}-\mathrm{S}$ is shown in Figure 3. These mixture velocities are compared to the numerical results of Shi at the test line located at $x=0$ and $z=100$ and $D=2.6 \mathrm{~m}$. The two velocity distributions give a small discrepancy with the Shi profile. The deformation of the axial velocity around the upper pipe wall is well reproduced. This deformation leads to a nonsymmetric character of these profiles and varies with the variation of the two-phase interface.

For the 3D-T configuration, the computed maximum axial velocity is $W_{\max }=1.56 \mathrm{~m} / \mathrm{s}$ which is close to the $\mathrm{Shi}$ 


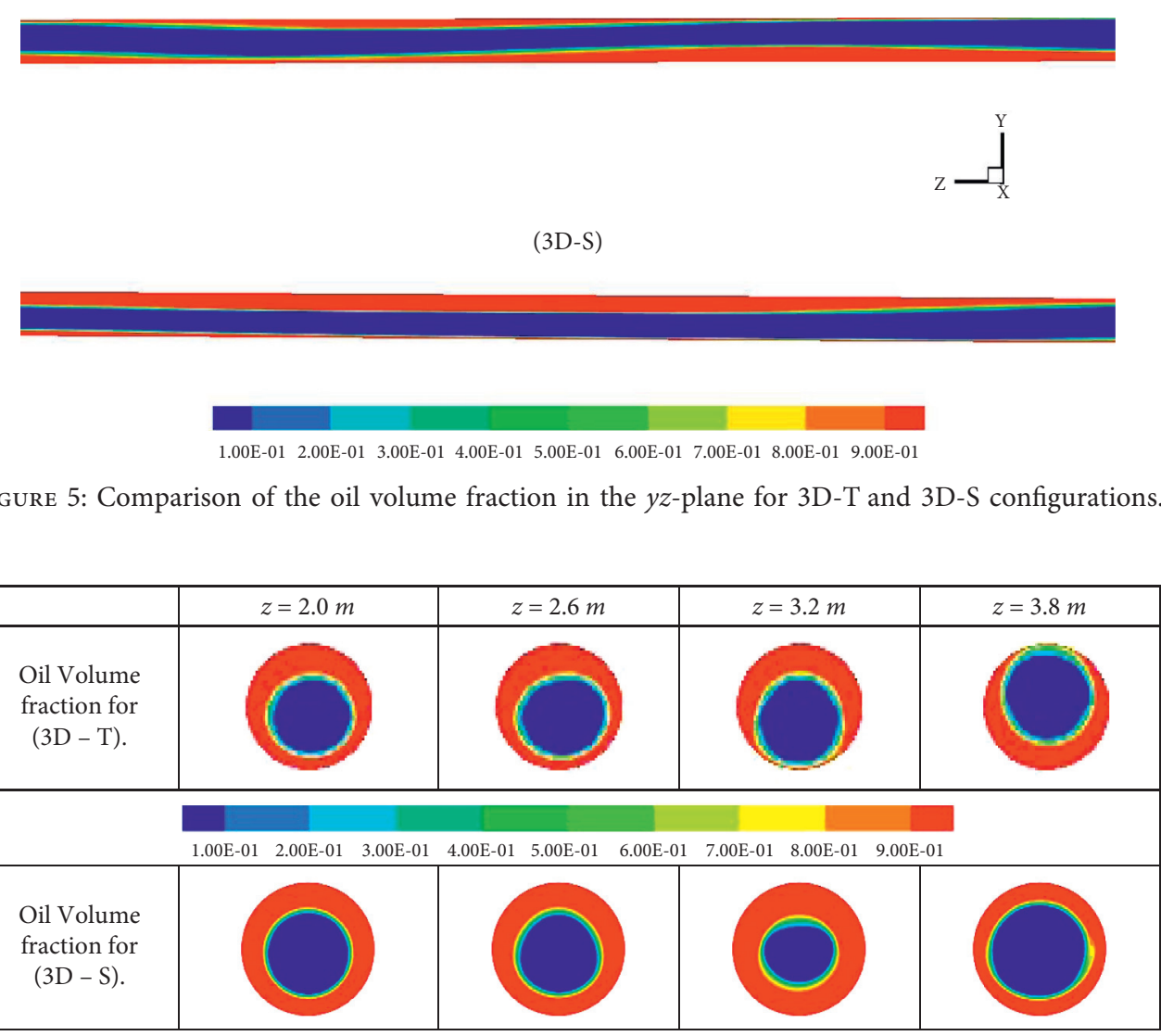

Figure 6: Comparison of the oil volume fraction in the $x y$-plane for 3D-T and 3D-S configurations.

\begin{tabular}{|c|c|c|c|c|c|}
\hline $\begin{array}{c}\text { Turbulent } \\
\text { kinetic } \\
\text { energy for } \\
(3 \mathrm{D}-\mathrm{T}) .\end{array}$ \\
\hline
\end{tabular}

Figure 7: Comparison of the turbulent kinetic energy in the $x y$-plane for 3D-T and 3D-S configurations.

value in [12] equal to $1.52 \mathrm{~m} / \mathrm{s}$. The $3 \mathrm{D}-\mathrm{S}$ slightly overestimates the maximum velocity which is equal to $1.63 \mathrm{~m} / \mathrm{s}$.

Figure 4 shows the simulated oil volume fraction distribution in the same line location $x=0$ and $z=100 \mathrm{D}$. The $\mathrm{CAF}$ regime is more concentric for the $3 \mathrm{D}-\mathrm{S}$. However, the oil volume fraction for the 3D-T approaches the Shi profile. In all simulated cases, the film water film thickness at the upper pipe wall is slightly greater than at the lower pipe wall. Conformed to Shi in [12], the CAF regime is more concentric when the inertial force is dominant.
The comparison of the oil volume fraction in the $y z$ plane for the two inlet configurations is represented in Figure 5. Each inlet configuration induces a different interaction between the oil and water phases. This results in different shapes of the CAF regime. The upper water film is thicker for the $3 \mathrm{D}-\mathrm{S}$, and this film is more irregular for 3D-T.

These observations are confirmed by representing the oil volume fraction in $x y$-plane for different locations along the pipe axis, as shown in Figure 6. The CAF for $3 \mathrm{D}-\mathrm{T}$ is more 


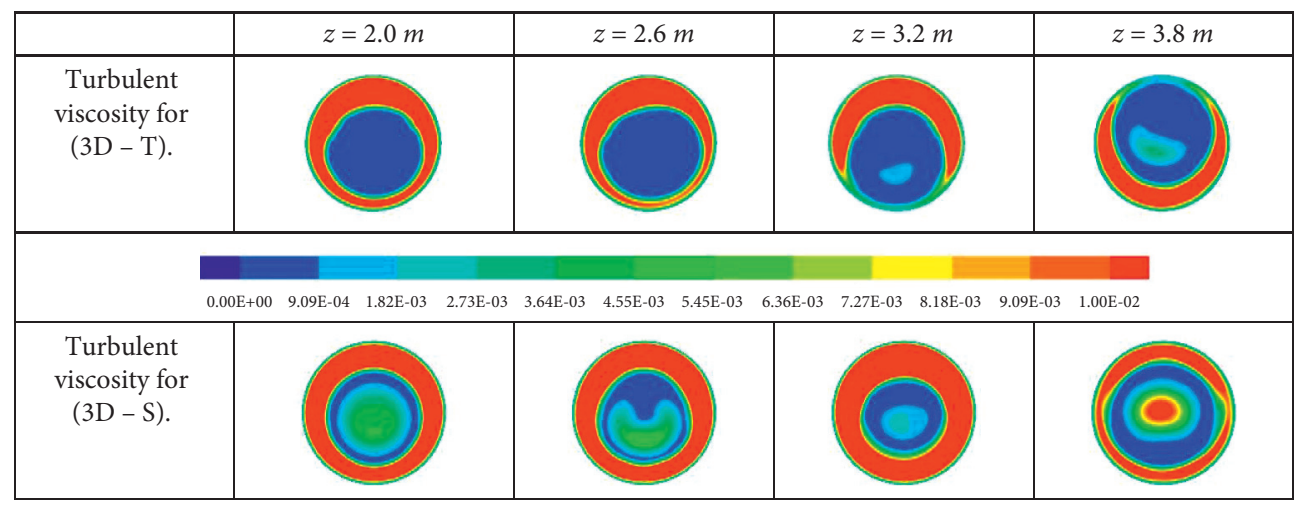

Figure 8: Comparison of the turbulent viscosity in the $x y$-plane for 3D-T and 3D-S configurations.

eccentric around the pipe axis especially in the downstream region of the horizontal pipe located away from $z>2.6 \mathrm{~m}$.

As confirmed by Shi simulations, the low turbulent kinetic energy $k$ is located at the core of the CAF and high $k$ located at the annular water region and close to the wall (Figure 7). The inlet configurations affect the distribution of $k$ due to the eccentricity of the CAF in 3D-T configuration.

The cross-section distributions of the turbulent kinetic energy for the CAF regime are represented in Figure 8. These distributions match the oil and water phase cross-section variations with a higher value in the water annular.

\section{Conclusions and Perspectives}

The AF regime of the liquid-liquid two-phase flow is validated for two inlet configurations at the horizontal and circular pipe. Compared to the experimental results of Charles et al. in [5] and numerical simulations of Shi in [12] good agreement was found in terms of pressure drop, oil volume fraction distribution, and axial velocity. To predict the interface deformation due to the interaction of the oil and water phase, the present model is based on the VOF convective transport equation coupled with the RANS equations and k-omega turbulence model.

This proposed model will be validated for the CAF regime with the high-viscosity oil-water flow, stratified, and slug flows. The large Eddy simulation (LES) can be used to better reproduce the small turbulence structure for this complex immiscible two-phase flow. As a future numerical investigation, we also propose a more complicated pipe configuration of the liquid-liquid flow such as those through an inclined or curved pipe. Finally, as a perspective of this study, we propose the development of a more sophisticated two-phase transport model taking into account the interface transfer between the liquid-liquid phases.

\section{Data Availability}

No data were used to support the study.

\section{Conflicts of Interest}

The authors declare that they have no conflicts of interest.

\section{Acknowledgments}

The authors gratefully acknowledge Qassim University, represented by the Deanship of Scientific Research, on the financial support for this research, under the no. 10133-cos2020-1-3-I, during the academic year 1442 AH/2020 AD.

\section{References}

[1] N. Brauner, "Two-phase liquid-liquid annular flow," International Journal of Multiphase Flow, vol. 17, no. 1, pp. 59-76, 1991.

[2] S. K. R. Cherlo, S. Kariveti, and S. Pushpavanam, "Experimental and numerical investigations of two-phase (Liquid-Liquid) flow behavior in rectangular microchannels," Industrial \& Engineering Chemistry Research, vol. 49, no. 2, pp. 893-899, 2010.

[3] P. Desir, T.-Y. Chen, M. Bracconi, B. Saha, M. Maestri, and D. G. Vlachos, "Experiments and computations of microfluidic liquid-liquid flow patterns," Reaction Chemistry \& Engineering, vol. 5, no. 1, pp. 39-50, 2020.

[4] M. A. Al-Yaari and B. F. Abu-Sharkh, "CFD prediction of stratified oil-water flow in a horizontal pipe," Asian Transactions on Engineering, vol. 1, no. 5, pp. 68-75, 2011.

[5] M. E. Charles, G. W. Govier, and G. W. Hodgson, "The horizontal pipeline flow of equal density oil-water mixtures," Canadian Journal of Chemical Engineering, vol. 39, no. 1, pp. 27-36, 1961.

[6] G. Elseth, An experimental study of oil/water flow in horizontal pipes, PhD Thesis, Norwegian University of Science and Technology, Trondheim, Norway, 2001.

[7] L. Amundsen, An experimental study of oil-water flow in horizontal and inclined pipes, $\mathrm{PhD}$ Thesis, Norwegian University of Science and Technology, Trondheim, Norway, 2011.

[8] T. Balakhrisna, S. Ghosh, G. Das, and P. K. Das, "Oil-water flows through sudden contraction and expansion in a horizontal pipe-phase distribution and pressure drop," International Journal of Multiphase Flow, vol. 36, no. 1, pp. 13-24, 2010.

[9] H. Al-Awadi, Multiphase characteristics of high viscosity oil, PhD Thesis, Cranfield University, Cranfield, UK, 2011.

[10] D. S. Santos, P. M. Faia, F. A. P. Garcia, and M. G. Rasteiro, "Oil/water stratified flow in a horizontal pipe: simulated and experimental studies using EIT," Journal of Petroleum Science and Engineering, vol. 174, pp. 1179-1193, 2019.

[11] X. Liu, C. Gong, L. Zhang, H. Jin, and C. Wang, "Numerical study of the hydrodynamic parameters influencing internal 
corrosion in pipelines for different elbow flow configurations," Engineering Applications of Computational Fluid Mechanics, vol. 14, no. 1, pp. 122-135, 2020.

[12] J. Shi, A study on high-viscosity oil-water two-phase flow in horizontal pipes, PhD Thesis, Cranfield University, Cranfield, UK, 2015.

[13] S. Al Jadidi, Lubricated transport of heavy oil investigated by $c f d, \mathrm{PhD}$ thesis, University of Leicester, Leicester, UK, 2017.

[14] S. A. Konings, Numerical Simulations of Core-Annular Flow through a Horizontal Pipe, Delft University of Technology, Delft, Netherlands, 2017.

[15] W. Jiang, J. Wu, S. Du, C. Liu, and Y. Liu, "CFD simulation on oil-water annular flow through CAF generator device," Energy Sources, Part A: Recovery, Utilization, and Environmental Effects, vol. 42, no. 15, pp. 1861-1873, 2020.

[16] N. Ayuba, R. B. Buhler, L. Silva da Silva, and T. Lopes, "Application of density-viscosity in predicting oil-water flow profile in horizontal pipe," Experimental and Computational Multiphase Flow, pp. 1-19, 2020, In press.

[17] J. Shi, M. Gourma, and H. Yeung, "CFD simulation of horizontal oil-water flow with matched density and medium viscosity ratio in different flow regimes," Journal of Petroleum Science and Engineering, vol. 151, pp. 373-383, 2017.

[18] D. C. Wilcox, "Formulation of the k-w turbulence model revisited," AIAA Journal, vol. 46, no. 11, pp. 2823-2838, 2008.

[19] J. U. Brackbill, D. B. Kothe, and C. Zemach, "A continuum method for modeling surface tension," Journal of Computational Physics, vol. 100, no. 2, pp. 335-354, 1992.

[20] H. Schlichting, Boundary-layer Theory, McGraw-Hill, New Yorkm NY, USA, 1979. 